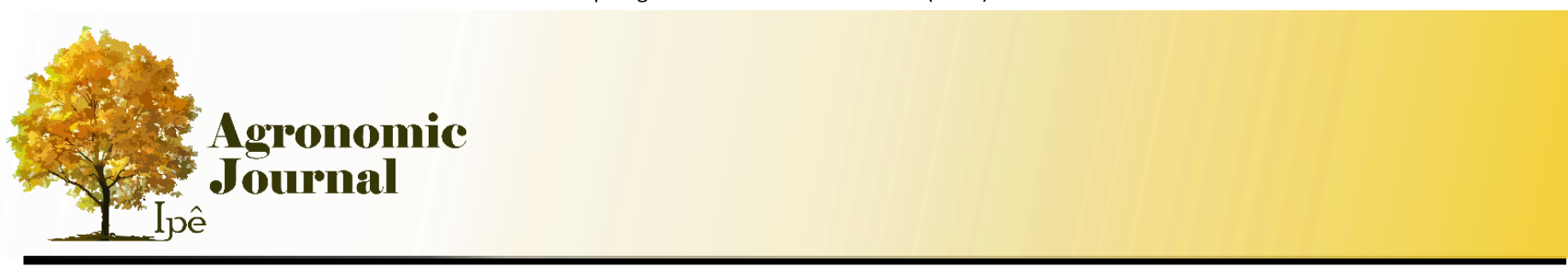

\title{
EFICÁCIA DE DOIS CONTROLES PREVENTIVOS PARA DOENÇAS RESPIRATÓRIAS DE BOVINOS CONFINADOS
}

\author{
EFFECTIVENESS OF TWO PREVENTIVE CONTROLS FOR CONFINED CATTLE RESPIRATORY DISEASES
}

Mateus Santana Silva ${ }^{1}$, Daniel Ferreira Caixeta ${ }^{2}$, Manoel Henrique Reis Oliveira ${ }^{3}$, Dyb Youssef Bittar ${ }^{2}$

${ }^{1}$ Engenheiros Agrônomos - Faculdade Evangélica de Goianésia;

${ }^{2}$ Engenheiro Agrônomo, Professor da Faculdade Evangélica de Goianésia

${ }^{3}$ Engenheiro Agrônomo, Professor Itégo

\section{Info}

Recebido: 09/2019

Publicado: $11 / 2019$

ISSN: 2595-6906

Palavras-Chave: $D B R$, Ruminantes

Produção

.Keywords: Inforce 03®,

CattleMaster 4®, Pneumonia.

\section{Resumo}

Os bovinos criados em sistema intensivo de confinamento possuem maior risco ao desenvolvimento de doenças respiratórias, devido serem expostos a fatores estressantes como o transporte, aglomeração e mistura de animais de diferentes origens, ambientes e variações climáticas. 0 objetivo do trabalho foi avaliar a viabilidade sanitária das vacinas Inforce $03^{\circledR}$ e CattleMaster $4^{\circledR}$ no controle preventivo de pneumonia em bovinos submetidos ao sistema intensivo de

engorda. O experimento foi conduzido na Fazenda Vera Cruz, do Grupo Otavio Lage, localizado as proximidades da GO 080, KM 65,5, município de Goianésia, estado de Goiás. Nos lotes foram avaliadas as seguintes vacinas, CattleMaster $4^{\circledR}$, Inforce 3 e COMBO (CattleMaster $4^{\circledR}+$ Inforce 3 ), utilizadas para prevenção da pneumonia. A vacina para pneumonia Inforce $03^{\circledR}$, promoveu maiores ganho de peso vivo, ganho médio diário, peso morto, peso morto em @, retorno financeiro por animal $(p<0,01 \%)$. Pensando no peso morto, ou seja, no rendimento dos animais em @ foi 15\% superior aos demais tratamentos, e promoveu um retorno de $\mathrm{R} \$ 141,03$ a mais em relação ao principal concorrente $(p<0,01 \%)$. A vacina Inforce $03^{\circledR}$ se destacou com os melhores resultados para para ganho de peso vivo, ganho médio diário, peso morto, peso morto em arrobas (@) e ganho em R\$ por animal. Em relação a morbidade dos lotes o melhor resultados foram obtidos com o COMBO e a Inforce $03^{\circledR}$.

\section{Resumo}

Cattle raised in intensive confinement systems are at greater risk for the development of respiratory diseases due to their exposure to stressors such as the transport, agglomeration and mixing of animals from different origins, environments and climatic variations. The objective of this study was to evaluate the sanitary viability of Inforce $03^{\circledR}$ and CattleMaster $4^{\circledR}$ vaccines in the preventive control of pneumonia in cattle submitted to intensive fattening system. The experiment was carried out at Fazenda Vera Cruz, from the Otavio Lage Group, located near GO 080, KM 65.5, Goianésia municipality, Goiás State. for prevention of pneumonia. One batch received only CattleMaster $4^{\circledR}$, and another batch vaccinated with Inforce $03^{\circledR}$, while a third batch received both CattleMaster $4^{\circledR}+$ Inforce $03^{\circledR}$ combo vaccines. The Inforce $03^{\circledR}$ pneumonia vaccine promoted higher liveweight gain, average daily gain, deadweight, deadweight in @, financial return per animal ( $p$ $<0.01 \%)$. Thinking about the dead weight, that is, the animal yield in @ was $15 \%$ higher than the other treatments, and promoted a return of $R \$ 141,03$ more than the main competitor ( $p<0,01 \%)$. The Inforce $03^{\circledR}$ vaccine stood out with the best results for live weight gain, average daily gain, dead weight, arrobas dead weight (@) and gain in R \$ per animal. Regarding batch morbidity, the best results were obtained with COMBO and Inforce $03^{\circledR}$. 


\section{INTRODUÇÃO}

Segundo CERQUEIRA, 2017 o Brasil contém o maior rebanho comercial de gado bovino no mundo, com mais de 217 milhões de cabeça, e ocupa a segunda posição mundial em abate de bovinos com mais de 42 milhões de cabeças abatidas, em 2014. Em relação a quantidade total de animais produzidos e abatidos, apenas 4,66 milhões de cabeças (11\% do abate total) foram terminadas em confinamento de bovinos no Brasil.

O confinamento de bovinos de corte é caracterizado como um sistema intensivo de produção de carne em larga escala, em uma pequena área e com alta quantidade, respeitando os aspectos sanitários, nutricionais, comportamentais dos animais e do meio ambiente (FILHO, 2011). O objetivo principal desse sistema de confinamento é produzir muito em pouco espaço e tempo, porém a alta taxa de lotação de bovinos apresenta um grande desafio no que diz respeito ao manejo sanitário (SOUZA et al., 2017).

Os bovinos criados em sistema intensivo de confinamento possuem maior risco ao desenvolvimento de doenças respiratórias (DBR) devido serem expostos a fatores estressantes como o transporte, aglomeração e mistura de animais de diferentes origens, ambientes e variações climáticas. (CERQUEIRA, 2017). Coutinho (2004) afirma que a incidência da DBR, ocorre nas três primeiras semanas de confinamento.

Em um confinamento comercial a qualidade do animal é de grande importância. As causas de morte em confinamento variam em função do desafio encontrado na região onde o mesmo é instalado. Em análise feita de 2014 a 2016, a pneumonia foi apontada como principais causas de mortalidade, seguida pela clostridioses e causas acidentais (BAPTISTA, 2017).

A DBR é uma doença infecciosa e com causas multifatoriais, que podem suprimir o sistema imune do hospedeiro, permitindo que vírus e bactérias rapidamente se repliquem no trato respiratório. O complexo das doenças respiratórias dos bovinos é decorrente do desequilíbrio entre as defesas naturais do animal e os fatores externos que favorecem a doença. (REZENDE, 2010).

A DBR aumenta os custos e consistem principalmente de custos diretos, representados pela morbidade, mortalidade, redução do crescimento animal e ganho de peso, impacto negativo sobre as características de carcaça, e os custos indiretos, relacionados à necessidade de infraestrutura adequada $\mathrm{e}$ aumento de manejo dos animais (CERQUEIRA, 2017).

No Brasil, os agentes patogênicos $M$. baemolytica, P. multocida e H. somni são os mais encontrados e de suma importância na morbidade e na mortalidade por doenças respiratórias no confinamento intensivo. Como estratégia preventivo para a DRB, compõe-se a profilaxia, em razão dos animais serem vacinados crescem os títulos de anticorpos, dessa forma reduz a ocorrência de doenças clínicas e melhora a performance da produção do confinamento (MAGALHÃES, 2017).

A vacinação e o método que se dá aos animais um modo eficiente de prevenção a uma determinada doença, assim como objetivo de reduzir ou banir um surto de doença respiratória, considerando um interesse quando se diz doença subclínica (SLOMPO et al., 2017).

A prevenção é o melhor método no controle da DRB. O manejo preventivo, e o manejo mais eficiente para minimizar a incidência e o grau de gravidade dos problemas respiratórios de um confinamento (Margarido et al., 2008). A utilização de vacinas tem sido a melhor técnica de prevenção e controle eficaz (SLOMPO et al., 2017), o uso de vacinas também é recomendado 45 dias antes da entrada no confinamento auxiliando no conjunto de práticas que auxilia no controle e prevenção das DRB (BAPTISTA, 2017). 
O objetivo do trabalho foi avaliar a viabilidade sanitária das vacinas Inforce $03^{\circledR} \mathrm{e}$ CattleMaster $4^{\circledR}$ no controle preventivo de pneumonia em bovinos submetidos ao sistema intensivo de engorda.

\section{MATERIAL E MÉTODOS}

O experimento foi conduzido na Fazenda Vera Cruz, do Grupo Otavio Lage, localizado as proximidades da GO 080, KM 65,5, município de Goianésia, estado de Goiás, com as seguintes coordenadas geográficas, $15^{\circ} 16^{\prime} 46.9^{\prime \prime} \mathrm{S}$ e $49^{\circ} 02^{\prime} 25.0^{\prime \prime}$ W.

O clima é do tipo Aw (segundo a classificação de Köppen-Geiger), caracterizado tropical com chuvas no verão, possuindo duas estações bem definidas. Precipitação pluvial média de $1502 \mathrm{~mm}$ anuais, e 24,4 C de temperatura média.

Foram avaliados três lotes de animais machos não castrados, das raças nelores e compostas, terminados no sistema de confinamento. A idade dos animais varia entre 22 a 29 meses de idade.

Nos lotes foram avaliadas as seguintes vacinas, CattleMaster $4^{\circledR}$, Inforce 3 , ambas para prevenção da pneumonia. Um dos lotes recebeu somente a CattleMaster $4^{\circledR}$, e outro lote vacinado com Inforce $03^{\circledR}$, já um terceiro lote recebeu as duas vacinas (combo) CattleMaster $4^{\circledR}+$ Inforce $03^{\circledR}$ (Tabela 1$)$.

Tabela 1 - Tratamentos

\begin{tabular}{lll}
\hline Localidade/Curral & Quant. de animais & Vacinas \\
\hline G-07 & 147 & Inforce 03 \\
G-08 & 150 & Combo \\
G-10 & 150 & Cattlemaster 4 ${ }^{\circledR}$ (Zoetis) \\
\hline
\end{tabular}

Os lotes foram separados de forma homogênea no primeiro dia de confinamento e levado aos currais de descanso que permaneceram por um período de três dias. Logo em seguida no quarto dia foram realizados o protocolo sanitário a realização das vacinas, vermifugação, pesagens, e identificação individual dos animais. Após todo o processo os animais entraram nos currais do confinamento.

Os animais foram sendo acompanhado do primeiro dia de confinamento até o abate. Todos os dias os lotes foram observados, durante um período médio de 100 dias. Todos os dias lotes eram acompanhados para a identificação de presença de animal doente. Os animais identificados com a algumas doenças eram medicado e registrado o número do animal para controle.

$\mathrm{Na}$ (tabela 2) foram avaliados 11 animais vacinados com CattleMaster $4^{\circledR}, 13$ animais com Combo, e 14 animais com Inforce $03^{\circledR}$ para a avaliação dos seguintes parâmetros, peso médio inicial, ganho de peso vivo, ganho médio diário, peso morto, peso morto em arrobas e ganho $\mathrm{R} \$$ por animal, todos os animais entraram com peso entre 390 a $400 \mathrm{~kg}$.

Foram avaliados animais com peso $5 \mathrm{~kg}$ acima e abaixo da média para retirar o efeito do peso médio dos lotes, feita essa prospecção, foi realizada a análise comparativa das principais concorrentes com todos os animais do lote.

Seguindo na (tabela 3), foram avaliados 120 animais vacinados com Inforce $03^{\circledR}$ e 130 animais com CattleMaster $4^{\circledR}$, sendo compradas peso médio inicial, ganho de peso vivo, ganho médio diário, peso morto, peso morto em arrobas e ganho $\mathrm{R} \$$ por animal.

Não seria possível satisfazer as bases da experimentação agrícola para comparar as diferentes vacinas, nem, tão pouco, lotes de animais. Assim, as variáveis analisadas foram comparadas por meio do teste de $\mathrm{X}^{2}$ (SAS Institute, 2004), que não faz nenhuma 
inferência quanto ao controle local ou casualização. Todos os contrastes possíveis foram ajustados manualmente.

\section{RESULTADOS E DISCUSSÃO}

Conforme pode ser observado na (tabela 2) abaixo os lotes entraram iguais, ou seja, qualquer variação observada ocorreu, provavelmente, em função do protocolo sanitário empregado ( $\mathrm{p}>0,05 \%)$.

A vacina para pneumonia Inforce $03^{\circledR}$, promoveu maiores ganho de peso vivo, ganho médio diário, peso morto, peso morto em @, retorno financeiro por animal $(\mathrm{p}<0,01 \%)$. Pensando no peso morto, ou seja, no rendimento dos animais em @ foi $15 \%$ superior aos demais tratamentos, e promoveu um retorno de $\mathrm{R} \$ 141,03$ a mais em relação ao principal concorrente $(p<0,01 \%)$.

A terapia metafilático com INFORCE $03^{\circledR} \mathrm{em}$ bovinos foi eficiente ao aumentar o ganho de peso vivo, ganho médio diário, peso morto, peso morto em @ e o retorno financeiro, quando comparada com bovinos tratados com CATTLE e COMBO (INFORCE + CATTLE).

A intensidade da reação vacinal e sua resposta no organismo está ligada a fatores de hipersensibilidade e isso depende de cada indivíduo (CEZAR, 2008). É provável que a superdosagem da vacina (COMBO) pode ter causado uma maior reação imunológica no animal, ocasionando febre e queda no apetite, pois as vacinas utilizadas no experimento, contém agente viral vivo (MLV) que ocasiona uma resposta mais rápida de três a cinco dias (BROCK et al., 2007), estimulando tanto a resposta imune humoral quanto a resposta imune celular (RIDPATH, 2013), no entanto, essas vacinas, contraditoriamente, podem levar a quadros de imunossupressão (MARTIN et al., 1981;KAEBERLE, 1983) o que pode ter prejudicado no desempenho durante o confinamento.
Arthington et al (2013) afirma que a vacinação contra patógenos de DRB pode prejudicar, pelo menos transitoriamente, características no desempenho de bovinos de corte relatando redução da relataram redução da eficiência alimentar e no GMD em novilhas vacinadas contra Mannheimia haemolytica comparado com novilhas não vacinadas. Rodrigues et al. (2015) relata que a administração das vacinas similares para novilhos de corte reduziu o consumo alimentar por 72 horas após a vacinação para DRB.

Conforme pode ser observado na (tabela 3) abaixo os lotes INFORCE e CAT'TLE, entraram com peso iniciais iguais, ou seja, A variação observada ocorreu, provavelmente, em função do protocolo sanitário empregado ( $\mathrm{p}>0,05 \%)$. Realmente, mesmo com lotes de 120 e 130 animais, há uma diferença expressiva entre Inforce $03^{\circledR}$ e Cattlemaster $4^{\circledR}$, para todas as variáveis avaliadas após a entrada dos animais $(\mathrm{p}<0,001 \%)$.

Para peso morto e rendimento financeiro, que são as variáveis mais importantes, o rendimento dos animais em @ vacinados com a Inforce $03^{\circledR}$ foi ainda maior, $17 \%$ superior ao principal concorrente, e promoveu um retorno de R\$149,07 em relação ao principal concorrente $(\mathrm{p}<0,001 \%)$. Se multiplicarmos o retorno unitário pelos 120 animais, a vacina promoveu um retorno de $\mathrm{R} \$ 17.888,4$ a mais do o lote com a CattleMaster $4^{\circledR}$.

Seguindo na (tabela 3) a vacina Inforce $03^{\circledR}$ mostra que tem suas vantagens sobre a concorrente se comparadas em valores como ilustra na tabela. A grande evolução está voltada no ganho financeiro, ou seja, no resultado quando comparadas a concorrente CattleMaster $4^{\circledR}$. Além dos valores financeiros os animais obtiveram valores melhores nos quesitos de ganho de peso vivo, ganho médio diário e em peso morto se compradas a CattleMaster $4^{\circledR}$. 
Tabela 2 - Peso médio Inicial (Kg de P. V), Ganho de peso vivo, Ganho de peso diário, peso morto, peso morto em @ e Ganho em R\$ por animal, submetidos ao confinamento sob diferentes tratamentos (CATTLEMASTER 4®, (COMBO) e INFORCE 03®).

\begin{tabular}{|c|c|c|c|c|c|c|}
\hline Tratamento & Peso médio Inicial (kg de P.V.) & Ganho de peso Vivo & $\begin{array}{l}\text { Ganho médio } \\
\text { diário }\end{array}$ & Peso morto & $\begin{array}{l}\text { Peso morto em } \\
\text { arrobas (@) }\end{array}$ & $\begin{array}{l}\text { Ganho em R\$por } \\
\text { animal }\end{array}$ \\
\hline COMBO & $394,38 \pm 0,88 \mathrm{a}$ & $154,69 \pm 6,33 \mathrm{~b}$ & $1,54 \pm 0,06 \mathrm{~b}$ & $87,09 \pm 3,56 \mathrm{~b}$ & $5,80 \pm 0,23 \mathrm{~b}$ & $889,45 \pm 36,42 \mathrm{~b}$ \\
\hline Cattlemaster $4^{\circledR}$ & $395,45 \pm 0,80 \mathrm{a}$ & $157,75 \pm 8,76 \mathrm{~b}$ & $1,57 \pm 0,08 \mathrm{~b}$ & $88,18 \pm 4,89 \mathrm{~b}$ & $5,87 \pm 0,32 \mathrm{~b}$ & $900,65 \pm 50,01 \mathrm{~b}$ \\
\hline Inforce $03^{\circledR}$ & $395,5 \pm 0,70 \mathrm{a}$ & $185,78 \pm 8,87 \mathrm{a}$ & $1,85 \pm 0,08 \mathrm{a}$ & $101,99 \pm 4,87 \mathrm{a}$ & $6,79 \pm 032 \mathrm{a}$ & $1041,68 \pm 49,76 \mathrm{a}$ \\
\hline $\mathrm{CV} \%$ & 0,71 & 18,90 & 18,90 & 18,37 & 18,37 & 18,37 \\
\hline
\end{tabular}

Letras diferentes na mesma coluna mostram que houve disparidade entre as vacinas pelo teste de $\mathrm{X}^{2}(\mathrm{p}<0,01 \%)$.

Tabela 3 - CattleMaster $4 \bowtie$ e Inforce $03 \circledR$, submetidos ao confinamento sob diferentes tratamentos.

\begin{tabular}{|c|c|c|c|c|c|c|}
\hline Tratamento & Peso médio Inicial (kg de P.V.) & Ganho de peso Vivo & $\begin{array}{l}\text { Ganho médio } \\
\text { diário }\end{array}$ & Peso morto & $\begin{array}{l}\text { Peso morto em } \\
\text { arrobas (@) }\end{array}$ & $\begin{array}{l}\text { Ganho em } R \$ \text { por } \\
\text { animal }\end{array}$ \\
\hline Cattlemaster $4^{\circledR}$ & $397,25 \pm 3,27 a$ & $152,58 \pm 2,39 \mathrm{~b}$ & $1,52 \pm 0,02 \mathrm{~b}$ & $85,29 \pm 1,33 b$ & $5,68 \pm 0,08 \mathrm{a}$ & $871,13 \pm 13,64 b$ \\
\hline Inforce $03^{\circledR}$ & $411,83 \pm 4,62 \mathrm{a}$ & $181,95 \pm 2,95 \mathrm{a}$ & $1,81 \pm 0,02 \mathrm{a}$ & $99,89 \pm 1,52 \mathrm{a}$ & $6,65 \pm 0,10 \mathrm{a}$ & $1020,20 \pm 16,58 \mathrm{a}$ \\
\hline CV \% & 11,06 & 19.92 & 19.92 & 19.52 & 19.52 & 19.52 \\
\hline
\end{tabular}

Letras diferentes na mesma coluna mostram que houve disparidade entre as vacinas pelo teste de $\mathrm{X}^{2}(\mathrm{p}<0,001 \%)$. 
$\mathrm{Na}$ tabela 03 podemos observar que os animais tratados com INFORCE $3^{\circledR}$ apresentaram um ganho de peso vivo de $181,95 \mathrm{Kg}$ contra $152,58 \mathrm{Kg}$ do lote Cattlemaster $4^{\circledR}$. Ao efetuarmos à análise econômica entre os lotes verificamos que os animais que receberam a vacina INFORCE $3^{\circledR}$ também apresentaram um ganho superior a Cattlemaster $4{ }^{\circledR}$ de $\mathrm{R} \$ 1020,20$ contra $\mathrm{R} \$ 871,13$ respectivamente por animal. Em comparação a Cattlemaster $4^{\circledR}$, a INFORCE $3{ }^{\circledR}$ resultou em um ganho de R\$149,07 a mais por animal.

Dos 447 animais avaliados, 14,76 apresentaram sintomatologia compatível com DRB. A morbidade das DRB no estudo foi de $10,66 \%$ (16/150) no tratamento $1,19,33 \%(29 / 150)$ no tratamento 2 e 14,28\% (21/147) no tratamento 3 (tabela 4). Animais submetidos ao protocolo metafilático 1 e 2 tiveram resultado semelhantes e sem diferença $(\mathrm{P}<0,001)$ entre si, e ambos, apresentaram índice de morbidade menor que o tratamento 3 com diferença estatística $(\mathrm{P}<0,001)$. Podemos observar que, a metafilaxia foi eficiente em reduzir os índices de morbidade em relação à DRB, estando de acordo com o objetivo da terapia profilática (SWEIGER \& NICHOLS, 2010). A metafilaxia reduziu os índices de morbidade, sobretudo em animais de alto risco para DRB, enquadrando-se nas recomendações abaixo de 10\% de APLEY (2015).

A avaliação visual de sinais clínicos da DRB é um método que padroniza a identificação da doença e não necessita de equipamentos ou ferramentas de alto custo (LOVE et al., 2014). Apesar de alguns autores considerarem este método de baixa sensibilidade e especificidade (AMRINE et al., 2014), o DART system neste estudo foi eficiente em identificar os animais doentes.

Tabela 4 - CATTLEMASTER 4® (COMBO) e INFORCE 03®, submetidos ao confinamento sob diferentes tratamentos.

\begin{tabular}{lll} 
Tratamento & Porcentagem de animais sadios $\mathbf{( \% )}$ & \\
\hline COMBO & 89,33 & $\mathrm{~A}$ \\
Inforce 03 $^{\circledR}$ & 85,71 & $\mathrm{AB}$ \\
Cattlemaster 4$^{\circledR}$ & 80,67 & $\mathrm{~B}$ \\
\hline
\end{tabular}

Letras diferentes na mesma coluna mostram que houve disparidade entre as vacinas pelo teste de $\mathrm{X}^{2}(\mathrm{p}<0,05 \%)$.

O tratamento que combina as duas vacinas só foi superior à CattleMaster $4^{\circledR}$ na porcentagem de animais que não foram tratados após a controle sanitário (rotina sanitária empregada na entrada dos lotes de animais no confinamento), ou seja, animais que não apresentaram sintomas de pneumonia ao longo do período de confinamento. Entretanto, a vacina Inforce não apresentou diferença na variável supracitada quando comparada à Cattle e Combo.

De forma geral, em trabalhos relatados sobre avaliações de DBR, os índices de morbidade encontrados neste estudo, foram superiores àqueles encontrados por BAPTISTA (2017), porém corrobam com os resultados de morbidade encontrados por Schneider et al., (2009) de 8,17\%, Fulton et al., (2009) de 14,7\% e Faber et al., (2000) de 20,7\%. Estes índices sugerem que as DRB são de importância tanto sanitária como econômica na indústria da carne brasileira, considerando os gastos de 14,3 milhões de dólares com mortalidade e 16,3 milhões de dólares com a morbidade associadas com DRB (BAPTISTA et al., 2016). 
Em grandes confinamentos a associação da vacinação e práticas de manejo profiláticos da mortalidade e da morbidade por DRB auxilia na redução dos desafios sanitários (WHITE, 2010). A utilização de antimicrobianos para redução de problemas respiratórios no período inicial de confinamento, objetiva o controle da doença aguda, devendo ser implantado em populações que tenham histórico de doenças ou não, visando a prevenção, sendo este, devendo ser realizado antes do início das mesmas (MAGALHÃES et al, 2017).

Schumaher (2019) relata que o adiantamento no momento da vacinação contra patógenos de DRB, ou seja, o fornecimento da vacina antes de se entrar no confinamento, pode mitigar a incidência de DRB e melhorar o desempenho dos bovinos no sistema de confinamento comercial.

Os manejos sanitários, contém várias vantagens como economia na produção e minimiza os custos dos tratamentos das doenças (FREITAS, 2012).

\section{CONCLUSÕES}

A vacina Inforce $03^{\circledR}$ se destacou obtivendo os melhores resultados para para ganho de peso vivo, ganho médio diário, peso morto, peso morto em arrobas (@) e ganho em R\$ por animal.

A vacina Inforce $03^{\circledR}$, promoveu um ganho por animal de $\mathrm{R} \$ 141,03$ a mais do que a Cattlemaster $4{ }^{\circledR}$ e ganho de $\mathrm{R} \$ 152,23$ a mais do que o COMBO.

Em relação a morbidade dos lotes o melhor resultados foram obtidos com o COMBO e a Inforce $03^{\circledR}$.

\section{REFERÊNCIAS BIBLIOGRÁFICAS}

ALMEIDA, E. A. O. Confinamento de bovinos para a produção de carne da linha Swift Black, JBS. Relatório de Estágio - Universidade Federal de Goiás, 2016.

AMRINE, D. E. et al. Pulmonary lesions and clinical disease response to Mannheimia haemolytica challenge 10 days following administration of tildipirosin or tulathromycin. Journal of Animal Science, v. 92, p. 311-319, 2014.

APLEY, M. Antimicrobial therapy of bovine respiratory disease. Veterinary Clinics of North America: Food Animal Practice, v. 13, n. 3, p. 549-574, 1997.

ARTHINGTON, J. D.; COOKE, R. F.; MADDOCK, T. D.; ARAUJO, D. B.; MORIEL, P.; DILORENZO, N.; LAMB, G. C. Effects of vaccination on the acutephase protein response and measures of performance in growing beef calves. Journal of Animal Science. v. 91, p. 1831-1837, 2013.

BAPTISTA, A. L.; REZENDE, A. L.; FONSECA, P. D. A.; NOGUEIRA, G. M.; HEADLEY, S. A.; MENEZES, G. L.; ALFIERI, A. A.; SAUT, J. P. E. Bovine respiratory disease complex associated mortality and morbidity rates in feedlot cattle from southeastern Brazil. Journal of Infection in Developing Countries, v. Submitted, 2016.

BAPTISTA, A. L; FONSECA, P. A; MENEZES, G. L; MAGALHÃES, L. Q. Doenças em bovinos confinados - desafios sanitários em um confinamento de grande porte. Revista Acad. Ciênc. Anim., v.15, p. 4 - 7, 2017.

BAPTISTA, ANDERSON LOPES. AVALIAÇÃO PRODUTIVA E SANITÁRIA EM BOVINOS CONFINADOS SOB METAFILAXIA ANTIMICROBIANA. Dissertação. Faculdade de Medicina Veterinária - UFU. Uberlândia MG - BRASIL. Janeiro - 2017.

BROCK, K. V; WIDEL, P.; WALZ, P.; WALZ, H. L. Onset of protection from experimental infection with type 2 bovine viral diarrhea virus following vaccination with a modified-live vaccine. v. $8, \mathrm{n}$. 1, p. 88,2007

CERQUEIRA, A.B. Doença respiratória em bovinos confinados: Aspectos patológicos e de desempenho produtivo. Dissertação Universidade Federal de Goiás, Programa de Pós-graduação em ciência animal, 2017.

FABER, R.; HARTWIG, N.; BUSBY, D.; BREDAHL, $\mathrm{R}$. The Costs and Predictive Factors of Bovine Respiratory Disease in Standardized Steer Tests. Beef Research Report, Publicação local, 2000.

FULTON, R. W. Host response to bovine viral diarrhea virus and interactions with infectious agents in the feedlot and breeding herd. Biologicals, v. 41, n. 1, p. 31-38, 2013. 
FILHO, A.D. Técnicas aplicadas para o confinamento de bovinos. Trabalho de conclusão de curso (graduação) - Universidade de Brasília, curso de medicina veterinária, 2011.

FREITAS, T. M. S.; Vacinas utilizadas no manejo sanitário de bovinos. Trabalho de conclusão de curso (graduação) - universidade federal de goiás escola de veterinária e zootecnia programa de pós-graduação em ciência animal, 2012.

LOVE, W. J. et al. Development of a novel clinical scoring system for on-farm diagnosis of bovine respiratory disease in pre-weaned dairy calves. PeerJ, v. 2, 2014.

KELLING, C. L.; HUNSAKER, B. D.; STEFFEN, D. J.; TOPLIFF, C. L.; ESKRIDGE, K. M. Characterization of protection against systemic infection and disease from experimental bovine viral diarrhea virus type 2 infection by use of a modified-live noncytopathic type 1 vaccine in calves. American Journal of Veterinary Research, v. 68, n. 7, p. 788-796, 2007

MAGALHÃES, L. Q. Eficácia de protocolos preventivos para doenças respiratórias dos bovinos confinados. Dissertação Universidade Federal de Uberlândia, 2017.

MAGALHÃES, L. Q.; BAPTISTA, A. L.; FONSCECA, P de A.; MENEZES, G. L.; NOGUEIRA, G. M.; HEADLEY, S, A.; FRITZEN, J. T. T.; ALFIERI, A. A.; SAUT, J. P. E.. Efeito do uso de protocolos metafiláticos segundo o risco de doença respiratória bovina em confinamentos. Ciência Rural, v. 47, n. 8, p. 1-6, 2017.

MARGARIDO, R. S.; NETO, L. D.; FERREIRA, F. V. Doenças respiratória dos bovinos. Revista Cientifica Eletrônica de Medicina Veterinária, jan. 2008.

MOURA, F. L. D. R. Avaliação da eficácia de uma vacina autógena de moraxella bovis no controlo da queratoconjuntivite infecciosa bovina em bovinos de carne. Dissertação - Universidade de Lisboa, 2017.

MARTIN, S. W.; MEEK, A. H.; DAVIS, D. G.; JOHNSON, R. A.; CURTIS, R. A. Factors associated with morbidity and mortality in feedlot calves: the Bruce County beef project, year two. Canadian. Journal of Comp Medicine Veterinary Science, v. 45, p. 103 112, 1981

REZENDE, M. L. G. Avaliação de parâmetros clínicos, hematológicos e viabilidade econômica do uso do Florfenicol na metafilaxia das afecções respiratórias inespecíficas de bovinos confinados no Estado de Goiás. Dissertação Universidade Federal de Goiás, 2010.

SAS INSTITUTE. SAS/STAT user'sguide, release 9.1 ed. SAS Institute, Cary, NC, 2004.

RIDPATH, J. Immunology of BVDV vaccines. Biologicals, v. 41, n. 1, p. 14-19, 2013.

RODRIGUES, M. C.; COOKE, R. F.; MARQUES, R. S.; CAPPELLOZZA, B. I.; ARISPE, S. A.; KEISLER, D. H.; BOHNERT, D. W. Effects of vaccination against respiratory pathogens on feed intake, metabolic and inflammatory responses in beef heifers. Journal of Animal Science. v. 93, p. 4443-4452. 2015.

SCHNEIDER, M. J.; TAIT, R. G., JR.; BUSBY, W. D.; REECY, J. M. An evaluation of bovine respiratory disease complex in feedlot cattle: Impact on performance and carcass traits using treatment records and lung lesion scores. Journal of Animal Science, v. 87, n. 5, p. 18217, 2009.

SCHUMAHER, THIAGO FAVARETO. EFEITOS EM DIFERENTES PERÍODOS DA VACINAÇÃO CONTRA PATÓGENOS RESPIRATÓRIOS NA PERFORMANCE, RESPOSTA DE ANTICORPOS E A SAÚDE DE BOVINOS CONFINADOS. Dissertação. Botucatu, 2019.

SOUZA, R.C; SOUZA, R.C; MENEZES, G.L. Manejo sanitário em confinamentos. Revista Acad. Ciênc. Anim., v.15, p.191-192, 2017.

SLOMPO, D; BERTAGNON, H.G; HORST, E. H; NEUMANN, N; MAREZE, J; SOUZA, A. M; JÚNIOR, E. S.S; GOLDONI, I; ASKELL, E.J. Manejo do complexo respiratório bovino em confinamento: Revisão. Revista PUBVET, v.11, p. 381-392, 2017.

SWEIGER, S. H.; NICHOLS, M. D. Control methods for bovine respiratory disease in stocker cattle. Veterinary Clinics of North America: Food Animal Practice, v. 26, n. 2, p. 261-71, 2010. 